\title{
A Piezoelectric Flexural Plate Wave (FPW) Bio-MEMS Sensor with Improved Molecular Mass Detection for Point-of-Care Diagnostics
}

\begin{abstract}
A piezoelectric FPW-sensor has been developed for a point of care device in this work. The BioMEMS FPW-sensor consists of an electrode configuration termed as an interdigital transducer (IDT) placed on a membrane. An input IDT excites and an output IDT detects the propagating acoustic waves through a PZT layer.

Design optimizations and fabrication improvements of the FPW-sensor led to significantly reduced attenuation of the wave signal and the damping of the propagating waves between the IDTs. The working principle of mass loading is shown using different low-viscous liquids. A densitydependent sensitivity of $-0.39 \mathrm{MHz} / \mathrm{g} / \mathrm{cm}^{3}$ was evaluated.

After the membrane was functionalized, the Bio-MEMS FPW-sensor was used to measure a specific chemokine in complex solution. By design improvements, the resolution was significantly increased from $0.7 \mathrm{~Hz} / \mathrm{nM}$ to $14 \mathrm{~Hz} / \mathrm{nM}$.
\end{abstract}

Keywords: Flexural plate wave (FPW), Bio-MEMS, point of care, multiplexing, RSV

https://doi.org/10.1515/cdbme-2019-0067

\section{Introduction}

The Respiratory Syncytial Virus (RSV) is responsible of a high rate of post-neonatal deaths. A fast and early diagnosis with accurate detection is vital for an effective treatment. Common diagnostics for the identification of unknown pathogens require large sample volumes and are time consuming. In this work, a Piezoelectric Flexural Plate Wave

${ }^{*}$ Corresponding author: Christian Walk: Fraunhofer Institute for Microelectronic Circuits and Systems (IMS), Finkenstrasse 61, 47057 Duisburg, Germany, christian.walk@ims.fraunhofer.de Matthias Wiemann, Michael Görtz, Jens Weidenmüller, Andreas Jupe: Fraunhofer IMS, Duisburg, Germany, Karsten Seidl: University of Duisburg-Essen, Dep. of Electronic Components and Circuits, Duisburg, Germany
(FPW) Bio-MEMS sensor has been developed. The detection is based on the frequency shift of a FPW membrane due to binding of an additional mass depending on the applied functionalisation treatment.

Several acoustic sensors are actually been studied for molecular detection, like the Shear Horizontal Surface Acoustic Wave (SH-SAW) and the Surface Transverse Wave (STW) [1-2]. However, these sensors usually operate at frequencies larger than $100 \mathrm{MHz}$, which increases the overall device costs due to substantially more complicated readout electronics. Flexural Plate Wave (FPW) sensors, however, operate at lower frequencies. Depending on the design of FPW-sensors, the wave phase velocity can be less than that of sound. This means, only little energy is dissipated into a testing liquid [2-3], so a high mass sensitivity can be achieved.

\section{Sensor Layout and Fabrication}

A piezoelectric Bio-MEMS FPW-sensor has been developed for a point of care device. The piezoelectric material consists of lead zirconate titanate $\left(\mathrm{PbZrTiO}_{3}, \mathrm{PZT}\right)$ as its piezoelectric effect is three to nine times larger than a typically used piezoelectric material such as $\mathrm{AlN}$ or $\mathrm{ZnO}$ [4].

The FPW-sensor consists of a particular electrode configuration termed as an interdigital transducer (IDT). Specifically, IDTs comprise two identical comb-like structures, where respective fingers are placed on the surface in an interleaved alternating manner. The input IDT, with the aid of underlying piezoelectric layer, is used for the excitation and the output IDT for the detection of the propagating acoustic waves.

In prior publications the results of a first design variant (design "1") were presented, which had an extra oxide layer between metal and PZT layer [5]. In this work, we introduce an improved design (design "2") which is shown in Figure 1. 
Here, an oxide layer between the metal layers and the PZT was removed in order to increase the coupling. An additional TiW layer is used for better adhesion between the Ti/TiN and PZT layer. By replacing the oxide with a TiW layer the suppression of electron drift is appropriate and allows for a high signal integrity. Hereby, one photolithographic layer could be removed as TiW can be etched together with the metal above.

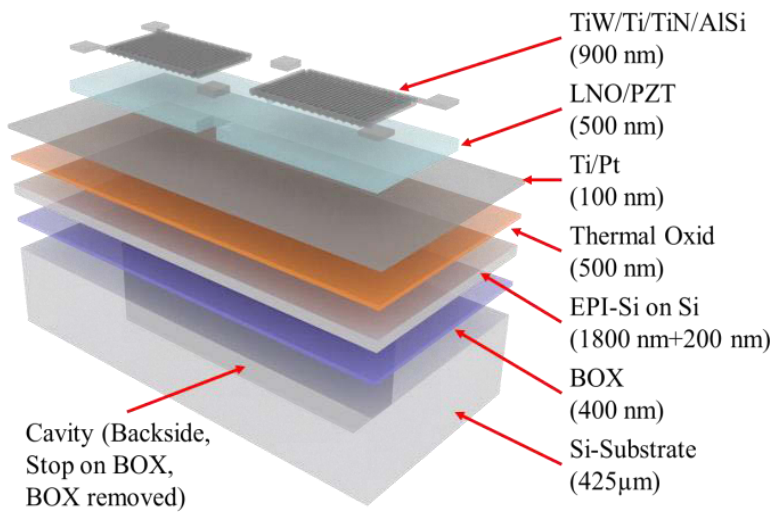

Figure 1: An exploded schematic of one Bio-MEMS FPW-sensor.

However, the more important reason for using the TiW layer was to improve the coupling of the waves between metal and PZT layer. The production process is described in more details in [5].

The acoustic waves theory behind the design of a BioMEMS FPW-sensor as well as the way to select its major geometrical parameters, such as drift length, pitch and fingers' length have been widely studied and reported [3].

Input and output IDTs are constructed by 17 pairs of AlSi fingers on a TiW barrier. The fingers width and length are $13.5 \mu \mathrm{m}$ and $320 \mu \mathrm{m}$, respectively. The pitch of the evaluated samples in this work is $44 \mu \mathrm{m}$ and drift length is $300 \mu \mathrm{m}$. The membrane of a Bio-MEMS FPW-sub-sensor consists of a $2-\mu \mathrm{m}$-thick epitaxial (EPI) grown $\mathrm{Si}$ on $\mathrm{Si}$, $500 \mathrm{~nm}$ thermal oxide, $100 \mathrm{~nm} \mathrm{Ti} / \mathrm{Pt}$ and $500 \mathrm{~nm} \mathrm{LNO/PZT}$. The total membrane thickness is thus $3.1 \mu \mathrm{m}$ over an area defined by the cavity of approx. $1.55 \times 0.34 \mathrm{~mm}^{2}$. According to simulation results a resonance frequency of about $16 \mathrm{MHz}$ can be expected in liquids with a density of about $1 \mathrm{~g} / \mathrm{cm}^{3}$.

The readout of the Bio-MEMS FPW-sensor is based on a network analyzer (NWA) used for excitation the PZT layer of the FPW-sub-sensor and recording its transmission spectrum. In this spectrum the most prominent resonance peak is identified by the NWA. The resonance frequency is monitored over time to detect any frequency shifts [6].

The assembling, packaging and connection for the sensor readout of the Bio-MEMS FPW-sensor to microfluidic interface is described in [7].

\section{Sensing mechanism of Bio- MEMS FPW-sensor}

In order to test the principle functionality of the Bio-MEMS FPW-sensor, four low-viscous liquids are applied. DI-Water, Isopropanol Alcohol (IPA), Tris buffer and saline solution $(4 \mathrm{M} \mathrm{NaCl})$ are used. Here, the viscosity effect is negligible in comparison with the mass loading effect [8]. The densities of the used liquids are listed in Table 1. Further, theoretical FPW-sensor considerations according to [3] led to the listed calculated sensor characteristics. The Bio-MEMS FPWsensor propagates and acoustic wave via a mechanical thin plate. The resonant frequency $f_{0}$ of a FPW-sensor membrane is given by eq. 1 ,

$$
f_{0}=\frac{v_{p}}{\lambda}
$$

where $\lambda$ is the acoustic wavelength and is the phase velocity. The phase velocity $v_{p, g}$ in air is given by eq. 2 ,

$$
v_{p, g}=\sqrt{\frac{A}{M}}
$$

where $A$ is the bending stiffness of a homogeneous plate,

$$
A=\left(\frac{\lambda}{2 \pi}\right)^{2} \frac{E \cdot d^{3}}{12\left(1-v^{2}\right)}
$$

and where $E$ is the Young's modulus, $v$ is the Poisson's ratio, $d$ is the membrane thickness. $\mathrm{M}$ is the mass per unit area of a homogeneous isotropic membrane plate.

The Bio-MEMS FPW-sensor is operated in liquid media. Thus, an additional stiffness effect is introduced by the liquids weight. The corrected phase velocity of the membrane under tensile stress and liquid loading, $v_{p, l}$, is given by eq. 4 ,

$$
v_{p, l}=\sqrt{\frac{T_{X}+A}{M+\rho_{l} \delta_{E}}}
$$

where $T_{X}$ is the component of in-plane tension in x-direction, $\rho_{l}$ is the density of the liquid, $\delta_{E}$ is the evanescent decay length. Because only low viscous liquids are considered in this work, any viscosity effects are neglected.

Table 1: Bio-MEMS FPW-sensor modelling for different liquids.

\begin{tabular}{lllll}
\hline & DI-Water & IPA & $\mathbf{4 ~ M ~ N a C l}$ & Tris \\
\hline Density in $\mathrm{g} / \mathrm{cm}^{3}$ & 0.9998 & 0.7860 & 1.1840 & 0.9996 \\
Phase velocity in m/s & 747.06 & 783.24 & 734.13 & 747.09 \\
Modelled resonant & 15.411 & 16.098 & 14.899 & 15.411 \\
frequency in $\mathrm{MHz}$ & & & & \\
\hline
\end{tabular}


In Figure 2, the shifts of the resonant frequency of a BioMEMS FPW-sensor are shown for injections of the lowviscous liquids. The sequence of injecting each $100 \mu 1$ of saline solution, IPA and DI-Water was applied twice.

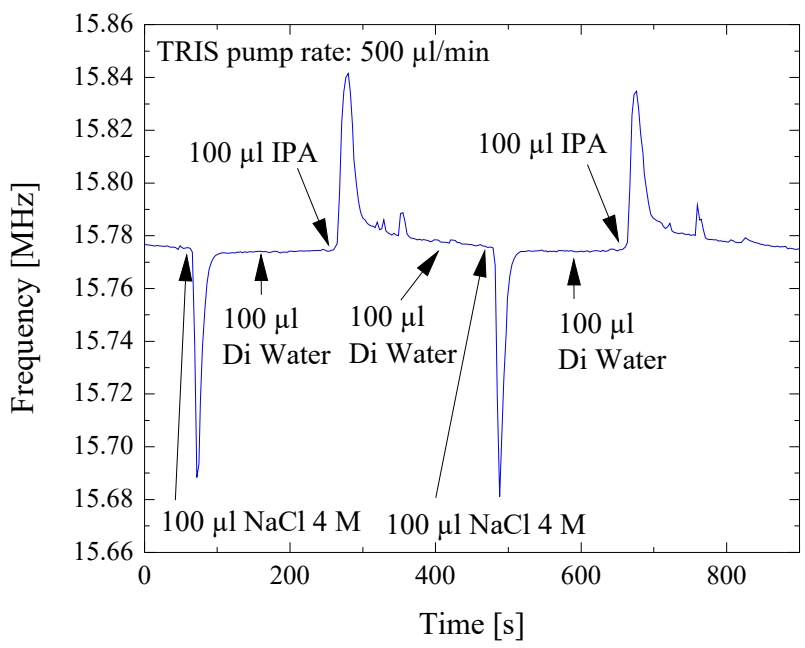

Figure 2: Response of a Bio-MEMS FPW-sensor after the injection of each $100 \mu \mathrm{l} 4 \mathrm{M} \mathrm{NaCl}$, Di-water and IPA in Tris.

In Figure 3 the resonant frequencies are plotted against the density. The sensitivity of the Bio-MEMS FPW-sensor is about $-0.39 \mathrm{MHz} / \mathrm{g} / \mathrm{cm}^{3}$ and has a linear regression coefficient $\mathrm{R}^{2}$ of 0.967 . The shown frequency shifts are evaluated and compared to the calculated resonant frequencies listed in Table 1.

The results obtained in this work are compared with a related FPW device [8]. It has a similar period of the IDT structures $(40 \mu \mathrm{m})$ and a similar total membrane thickness $(2.3 \mu \mathrm{m})$. The area of the membrane, however, is much larger compared to this work $\left(11.34 \mathrm{~mm}^{2}\right.$ vs. $\left.0.53 \mathrm{~mm}^{2}\right)$. Thus, this FPW device is most likely less affected by microfluidic coupling issues. In this work, the liquid injection is dynamical, whereas the liquid load is applied statically in [8]. In Figure 4, the results of both works are plotted with calculated and measured frequency shifts. The calculated sensitivity of the reference FPW device is $-0.85 \mathrm{MHz} / \mathrm{g} / \mathrm{cm}^{3}\left(\mathrm{R}^{2}=0.998\right)$ and the measured sensitivity is about $-0.60 \mathrm{MHz} / \mathrm{g} / \mathrm{cm}^{3}\left(\mathrm{R}^{2}=0.813\right)$.

The comparison of both FPW-sensors shows that the Sensor of this work is designed to show a much higher sensitivity. While the referred FPW device shows a relatively good agreement to the model, the here presented Bio-MEMS FPW-sensor shows a deviation by a factor of 7.7. A potential reason may be the microfluidic. Injected liquids may not completely fill the cavity upon a Bio-MEMS FPW-sensor membrane, thus replacing the prior liquid only partially.
Then, the change in density can become much smaller than theoretically assumed.

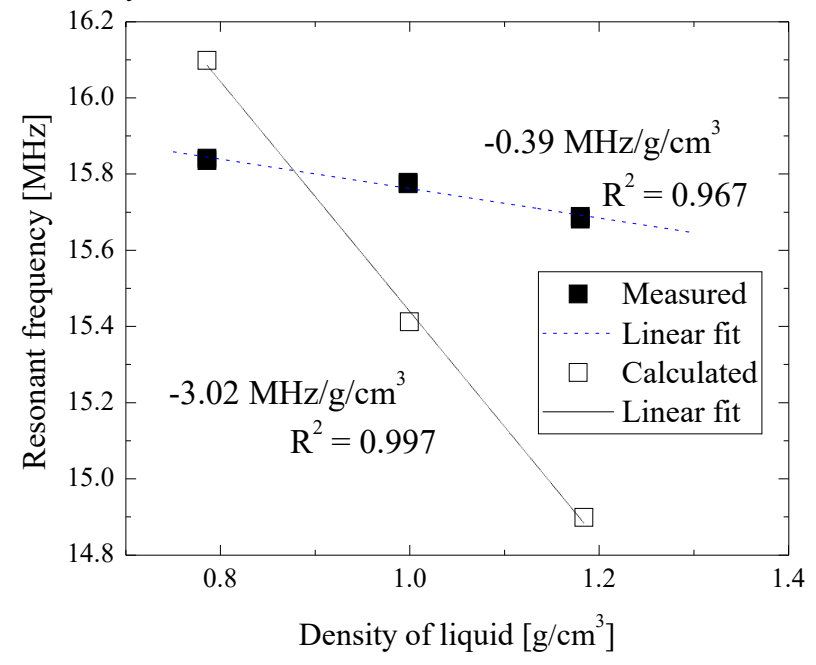

Figure 3: Sensitivity analysis of the calculated and measured resonant frequencies of a Bio-MEMS FPW-sensor and the density of low-viscosity liquids for a pump rate of $500 \mu \mathrm{l} / \mathrm{min}$.

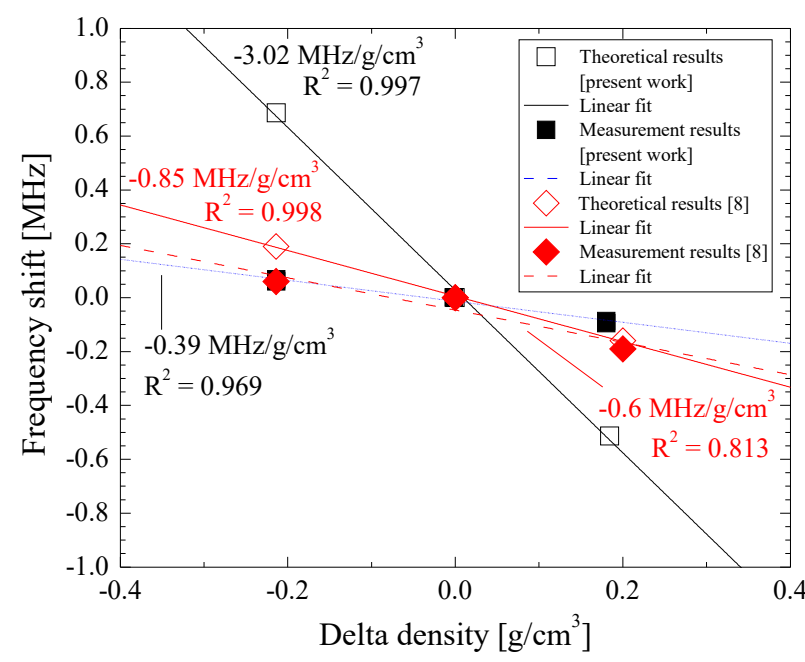

Figure 4: Comparison of the calculated and measured resonant frequencies of a Bio-MEMS FPW-sensor in this work to a FPW-device in [8]

\section{Results of functionalized Bio- MEMS FPW-sensor}

For the specific detection of target molecules such as chemokines, capture molecules are immobilized on the backside of the Bio-MEMS FPW-sensor membrane [5]. The Bio-MEMS FPW-sensor is mounted onto a microfluidic carrier which allows a liquid to flow from the input connection (fluidic input) passing over eight Bio-MEMS FPW-sensors integrated on a single chip to two exhaust 
connections [6]. A liquid of Tris buffer was continuously pumped along the sensor using a syringe pump. A 30- $\mu 1-$ bolus of Tris containing $500 \mathrm{nM}$ chemokine is added with a valve with added to the Tris buffer stream.

In design " 1 " an extra oxide layer separates the top TiW/Ti/TiN/AlSi metal from the about $1-\mu \mathrm{m}$-thick piezoelectric LNO/PZT layer [5]. Moreover, a pitch of $50 \mu \mathrm{m}$ and a drift length of $400 \mu \mathrm{m}$ were realized for the IDTs. A shift of about $350 \mathrm{~Hz}$ was detected after the injection of $500 \mathrm{nM}$ chemokine with a pump rate of $20 \mu \mathrm{l} / \mathrm{min}$.

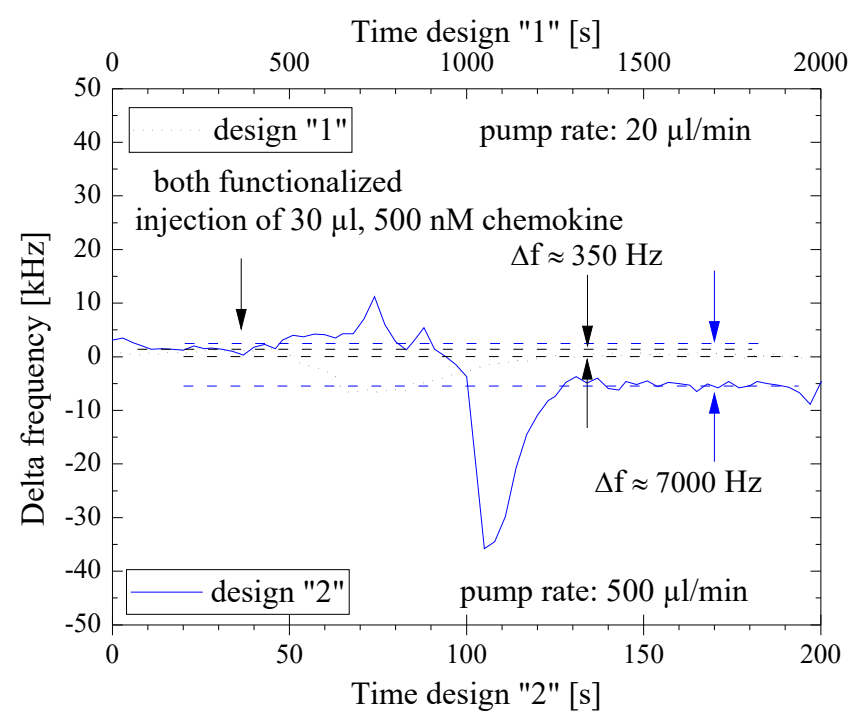

Figure 5: Frequency responses of functionalized Bio-MEMS FPW-sensor designs "1" and "2" after injections of $500 \mathrm{nM}$ chemokine buffered in Tris.

The design "2" was realized without an oxide layer between the top TiW/Ti/TiN/AlSi metal and the LNO/PZT layer. Furthermore, the LNO/PZT thickness is reduced to $500 \mathrm{~nm}$ and the pitch, drift length of the IDTs are $44 \mu \mathrm{m}$ and $300 \mu \mathrm{m}$, respectively. For the design " 2 " a shift of about $7 \mathrm{kHz}$ is measured after injecting $500 \mathrm{nM}$ chemokine in Tris with a pump rate of $500 \mu \mathrm{l} / \mathrm{min}$ (Figure 5). The improvements resulted in a resolution of about $14 \mathrm{~Hz} / \mathrm{nM}$ chemokine. According to Biacore SPR signal analyses, the chemokine mass-density is in the magnitude of $100 \mathrm{pg} / \mathrm{mm}^{2}$ for the used concentration. Hence, a mass-sensitivity of the Bio-MEMS FPW-sensor in liquid of about $70 \mathrm{~Hz} / \mathrm{pg} / \mathrm{mm}^{2}$ was achieved.

\section{Conclusion}

Bio-MEMS FPW-sensors were successfully fabricated and tested. A resolution of about $14 \mathrm{~Hz} / \mathrm{nM}$ chemokine and an equivalent mass sensitivity of at least $70 \mathrm{~Hz} / \mathrm{pg} / \mathrm{mm}^{2}$ was achieved. Future work will include improvements regarding the fluid dynamics in order to improve the sensor signal. The experiments will be extended in order to characterise the sensor signal for reduced concentrations of the chemokine.

\section{Author Statement}

The PoC-ID project has received funding from the European Union's Horizon 2020 research and innovation program under grant agreement no. 634415.

This publication is partly funded by the Federal Ministry of Education and Research under the project reference nos. 16FMD01K, 16FMD02 and 16FMD03.

\section{References}

[1] M.I. Rocha-Gaso, C. March-Iborra, A. Montoya-Baides, A. Arnau-Vives, "Surface generated acoustic wave biosensors for the detection of pathogens: a review", Sensors 9, vol. 7, pp. 5740-5769, 2009

[2] I.Y. Huang, M.C. Lee, "Development of a FPW allergy biosensor for human IgE detection by MEMS and cystamine-based SAM technologies", Sens Actuators B, vol. 132, pp. 340-348, 2018

[3] Jr. D.S. Ballantine, R.M. White, S.J. Martin, A.J. Ricco, G.C. Frye, E.T. Zellers, H. Wohtjec, Acoustic wave sensors: theory, design and physiochemical application, Academic, New York, 1997

[4] P. Muralt, "Piezoelectic thin films for MEMS", Integrated Ferroelectrics, vol. 17, pp. 297-307, 1997

[5] A. Jupe, P. Livshits, S. Kahnert, M. Figge, S. Mross, M. Goertz, H. Kappert, H. Vogt, A. Goehlich, "Development of a Piezoelectric Flexural Plate-Wave (FPW) BioMEMS-Sensor for Rapid Point-of-Care Diagnostics" in Nanostructured Materials for the Detection of CBRN. NATO Science for Peace and Security Series A: Chemistry and Biology Conference, J. Bonča, S. Kruchinin (eds), pp. 199-212, 2018

[6] M. Wiemann, C. Walk, J. Weidenmüller, A. Jupe and K. Seidl. "Development of a Multi Channel Piezoelectric Flexural Plate Wave BIO-MEMS-Sensor for Rapid Point-of-Care Diagnostics" in Transducers 2019/Eurosensors XXXIII Conf. Proc., Berlin, Jun. 2019

[7] P. Reinecke, M.-T. Putze, L. Georgi, R. Kahle, D. Kaiser, D. Hueger, P. Livshits, J. Weidenmueller, T. Weimann, A. Turchanin, T. Braun, K.-F. Becker, M. Schneider-Ramelow, and K.-D. Lang, "Scalable hybrid microelectronic-microfluidic integration of highly sensitive biosensors", Int. Symposium on Microelectronics, pp.672-679, 2018

[8] Jyh-Cheng Yu and Huang-Yao Lin, "Sensing liquid density using resonant flexural plate wave devices with sol-gel PZT thin films", Microsyst Technol, vol. 14, pp. 1073-1079, 2008 